\title{
Synchronization of the Neural Response to Noisy Periodic Synaptic Input
}

\author{
A. N. Burkitt and G. M. Clark \\ The Bionic Ear Institute, 384-388 Albert Street, \\ East Melbourne, Vic 3002, Australia \\ E-mail: a.burkitt@medoto.unimelb.edu.au \\ g.clark@medodo.unimelb.edu.au
}

\begin{abstract}
The relationship between the timing of the synaptic inputs and the output spikes of leaky integrate and fire neurons with noisy periodic synaptic input is addressed using the recently developed integrated-input technique. The conditional output spike density in response to noisy periodic input is evaluated as a function of the initial phase of the inputs. This enables the phase transition matrix to be calculated, which relates the phase at which the output spike is generated to the initial phase of the inputs. The interspike interval histogram and the period histogram for the neural response to ongoing periodic mput are then evaluated by using the leading eigenvector of this phase transition matrix. The dependence of the synchronization index of the neural response upon the number and amplitude of synaptic inputs, the membrane time constant, the average rate of inputs and their frequency of modulation is examined.
\end{abstract}

\section{Introduction}

The temporal information contained in the responses of neurons plays an important role in a neuronal information processing in a number of different parts of the central nervous system (CNS). In auditory processing the degree of phase locking in the neural response to noisy periodic input plays an important role, where studies indicate that spikes in the auditory pathway are phase locked up to frequencies around $3-5 \mathrm{kHz}$ in mammals [21], [13] and up to frequencies of $8 \mathrm{kHz}$ in the Barn Owl [17]. Likewise, a similar phase-locked response is observed in electrosensory systems of a number of fish [2]. This pluase locked response has also been postulated to play a central role in temporal coding in the brain, where it may be used in feature linking and patteru segmentation [28], [9], [12]. For reviews of these and other instances of temporal information processing within the CNS see [25], [7].
In this study we investigate the relationship between the timing of noisy periodic synaptic inputs and the output spikes that are generated in leaky integrate and fire neurons. A recent investigation of this problem [15] examined the input and output rates over the range of input vector strengths [11] (also called the synchronization index) as well as identifying the conditions under which a neuron can art as a coincidence detector and thus convert a temporal code into a rate code. Their results show how the output rate depends upon the neural parameters, such as the number of synapses and the time course of the postsynaptic response to the inputs, as well as upon the input statistics [15]. They identify two parameters, namely the coherence gain and the quality factor for coincidence detection, which largely characterize the performance of a coincidence detector, and they plot these quantities for representative values of the neural parameters over the range of input vector strengths. However, since their analysis concerns only the output rate, they are not able to predict quantities that depend upon the details of the timing of individual output spikes.

Our study provides a significant extension of these results, in which we relate the time-distribution of the output spikes to the phase of the synaptic input, and thus calculate the synchronization index of the output spikes and their phase histogran as a function of the frequency and synchronization of the input. In order to carry out the analysis we make the approximation that the amplitude of the postsynaptic response to an individual input spike is small, which is equivalent to the diffusion approximation [27]. For neurons with large numbers of small amplitude inputs this approximation proves to be very accurate.

\section{Methods}

The relationship between the timing of the synaptic inputs and the output spikes requires an analysis of 
the interspike interval (ISI) distribution of the output spikes, which is obtained using our recently developed integrated-input technique [3], [4], [5]. The membrane potential, $V(t)$, is assumed to be reset to its initial value at time $t=0, V(0)=v_{0}$ after a spike (action potential, AP) has been generated. The membrane potential is the sum of the excitatory and inhibitory postsynaptic potentials (EPSPs and IPSPs respectively)

$$
\begin{aligned}
& V(t)=v_{0}+\sum_{k=1}^{N} a_{k} s_{k}(t) \\
& s_{k}(t)=\sum_{m=1}^{\infty} u_{k}\left(t-t_{k_{m}}\right)
\end{aligned}
$$

where the index $k=1, \ldots, N$ denotes the afferent fiber and the index $m$ denotes the $m^{\text {th }}$ input from the particular fiber, whose time of arrival is $t_{k_{m}}\left(0<t_{k_{1}}<\right.$ $\left.t_{k_{2}}<\ldots<t_{k_{m}}<\ldots\right)$. The amplitude of the inputs is taken to be a constant, $a$, which is positive for EPSPs and negative for IPSPs. The time course of an input at the site of spike generation is described by the synaptic response function $u_{k}(t)$ for the leaky integrate and fire model

$$
u_{k}(t)= \begin{cases}e^{-t / \tau} & \text { for } t \geq 0 \\ 0 & \text { for } t<0\end{cases}
$$

where $r$ is the time constant of the membrane potential decay. Consequently the membrane potential has a discontinuous jump of amplitude $a_{k}$ upon the arrival of an EPSP and then decays exponentially between inputs. The decay of the EPSP across the membrane means that the contribution from EPSPs that arrive earlier have partially decayed by the time that later EPSPs arrive.

The time-dependent rate of arrival of input spikes at a synapse is taken to be periodic with frequency $\omega$ and initial phase $\phi$, i.e., the phase of the input at the time when the summation commences,

$$
\lambda(t)=\bar{\lambda}_{\text {in }}\left(1+2 r_{\text {in }} \cos (\omega t+\phi)\right), 0 \leq r_{\text {in }} \leq 0.5,
$$

where $\bar{\lambda}_{\text {in }}$ is the time-averaged input rate on a single fiber and $r_{\text {in }}$ is the synchronization index of the input spikes, with $r_{\text {in }}=0.5$ representing a highly modulated input and values of $r_{\text {in }}$ closer to zero representing inputs that contain less of the frequency dependent component. This input rate represents an inhomogeneous (i.e., nonstationary) Poisson process [18].

The probability density of the potential $V(t)$ is denoted by $p\left(v, t \mid v_{0} ; \omega, \phi\right)$, which is the probability that the potential has the value $v$ at time $t$, given that the previous spike occurred at time $t=0$ when the potential is reset to the value $v_{0}$, the frequency of the input is $\omega$ and the initial phase is $\phi$. This probability density is given by the following normalized expectation value, which is the integrated average over the time-distribution of synaptic inputs [3], [4],

$$
p\left(v, t \mid v_{0} ; \omega, \phi\right)=\mathrm{E}\{\delta(V(t)-v)\}
$$

where $\delta(x)$ is the Dirac delta function and the expectation value is over the random time of arrival of the synaptic inputs. The frequency and phase dependence of the probability density arises through this averaging over the arrival times of the synaptic input, which is described here explicitly by $\lambda(t)$, equation(3). I Ising the Fourier integral representation of the Dirac delta function, the probability density may be written as

$$
\begin{aligned}
& p\left(v, t \mid v_{0} ; \omega, \phi\right) \\
& =\int_{-\infty}^{\infty} \frac{d x}{2 \pi} \exp \left\{i x\left(v-v_{0}\right)\right\} \times \\
& \operatorname{E}\left\{\exp \left(-i x \sum_{k=1}^{N} a_{k} s_{k}(t)\right)\right\} \\
& =\int_{-\infty}^{\infty} \frac{d x}{2 \pi} \exp \left\{i x\left(v-v_{0}\right)\right\} \prod_{k=1}^{N} F_{k}(x, t ; \omega, \phi) \\
& =\int_{-\infty}^{\infty} \frac{d x}{2 \pi} \exp \left\{i x\left(v-v_{0}\right) \sum_{k=1}^{N} \ln F_{k}(x, t ; \omega, \phi)\right\}
\end{aligned}
$$

where the function $F_{k}(x, t ; \omega, \phi)$ is given by

$$
F_{k}(x, t ; \omega, \phi)=\mathrm{E}\left\{\exp \left(-i x a_{k} s_{k}(t)\right)\right\},
$$

and we have used the independence of the contributions from each fiber to obtain the product of $F_{k}$ 's in equation(5). Expanding the exponential in powers of $a_{k}$ we obtain

$$
\begin{aligned}
\ln F_{k}(x, t ; \omega, \phi) \\
=\ln \mathrm{E}\left\{1-i x a_{k} s_{k}(t)-\frac{x^{2} a_{k}^{2}}{2} s_{k}^{2}(t)+O\left(a_{k}^{3}\right)\right\} \\
=\ln \left[1-i x a_{k} \mathrm{E}\left\{s_{k}(t)\right\}\right. \\
\left.\quad-\frac{x^{2} a_{k}^{2}}{2} \mathrm{E}\left\{s_{k}^{2}(t)\right\}+O\left(a_{k}^{3}\right)\right] \\
=\quad-i x a_{k} \mathrm{E}\left\{s_{k}(t)\right\} \\
\quad-\frac{x^{2} a_{k}^{2}}{2}\left[\mathrm{E}\left\{s_{k}^{2}(t)\right\}-\left(\mathrm{E}\left\{s_{k}(t)\right\}\right)^{2}\right]+O\left(a_{k}^{3}\right),
\end{aligned}
$$

where $O(y)$ is a quantity that vanishes at least as fast as $y$ when $y \rightarrow \infty$. We henceforth neglect the $O\left(a_{k}^{3}\right)$ terms and retain only the linear and quadratic terms. This expansion to second order in powers of $a_{k}$ is an approximation which allows the resulting equations to be solved formally, and we do not address questions such 
as the convergence of the expansion. We expect the approximation to be valid for amplitudes of the postsynaptic potential that are small in comparison to the threshold.

The probability density function is evaluated in this Gaussian approximation as

$$
\begin{aligned}
& p\left(v, t \mid v_{0} ; \omega, \phi\right) \\
& =\int_{-\infty}^{\infty} \frac{d x}{2 \pi} \exp \left\{i x\left(v-v_{0}-\Upsilon(t ; \omega, \phi)\right)\right. \\
& \left.-\frac{x^{2}}{2} \Gamma(t ; \omega, \phi)\right\} \\
& =\frac{1}{\sqrt{2 \pi \Gamma(t ; \omega, \phi)}} \exp \left\{-\frac{\left(v-v_{0}-\Upsilon(t ; \omega, \phi)\right)^{2}}{2 \Gamma(t ; \omega, \phi)}\right\},
\end{aligned}
$$

with

$$
\begin{aligned}
\Upsilon(t ; \omega, \phi) & =\sum_{k=1}^{N} a_{k} \mathrm{E}\left\{s_{k}(t)\right\} \\
\Gamma(t ; \omega, \phi) & =\sum_{k=1}^{N} a_{k}^{2}\left(\mathrm{E}\left\{s_{k}^{2}(t)\right\}-\left(\mathrm{E}\left\{s_{k}(t)\right\}\right)^{2}\right)
\end{aligned}
$$

These expressions allow inputs with arbitrary amplitude distributions, including inhibitory inputs, to be considered. However, for simplicity we consider here the situation where the postsynaptic potentials from the inputs are all excitatory and equal in both amplitude, $a_{k}=a>0$, and time course, $u_{k}(t)=u(t)$, and where the rates on each of the $N$ afferent fibers are identical, $\lambda_{k}(t)=\lambda(t)$. Consequently we drop the index $k$, since all input fiber characteristics are identical. In the case of an inhomogeneous Poisson process with rate $\lambda(t)$ on each fibre, the expressions for $\Upsilon(t ; \omega, \phi)$ and $\Gamma(t ; \omega, \phi)$ take the forms [18]

$$
\begin{aligned}
\Upsilon(t ; \omega, \phi) & =N a \mathrm{E}\{s(t)\} \\
& =N a \int_{0}^{t} \lambda\left(t^{\prime}\right) u\left(t-t^{\prime}\right) d t^{\prime} \\
\Gamma(t ; \omega, \phi) & =N a^{2}\left(\mathrm{E}\left\{s^{2}(t)\right\}-(\mathrm{E}\{s(t)\})^{2}\right) \\
& =N a^{2} \int_{0}^{t} \lambda\left(t^{\prime}\right) u^{2}\left(t-t^{\prime}\right) d t^{\prime}
\end{aligned}
$$

The dependence of the expressions for $\Upsilon(t ; \omega, \phi)$ and $\Gamma(t ; \omega, \phi)$ above upon frequency and initial phase is therefore given explicitly througl the dependence upon $\lambda(t)$ (refractory effects are ignored here).

In order to calculate the probability density of output spikes it is necessary to find the time at which a spike is generated, namely the time at which the summed membrane potential crosses the threshold, $V_{\mathrm{th}}=v_{0}+\theta$, for the first time (called the first-passage time). The conditional first-passage time to threshold density, $f_{\theta}(t ; \omega, \phi)$, which depends upon the frequency, $\omega$, and phase, $\phi$, of the synaptic input is obtained from the integral equation [20], [3] (for $v \geq V_{\text {th }}$ )

$$
\begin{aligned}
& p\left(v, t \mid v_{0} ; \omega, \phi\right) \\
& =\int_{0}^{t} d t^{\prime} f_{\theta}\left(t^{\prime} ; \omega, \phi\right) p\left(v, t \mid V_{\mathrm{th}}, t^{\prime}, v_{0}\right)
\end{aligned}
$$

where $p\left(v_{2}, t_{2} \mid v_{1}, t_{1}, v_{0}\right)$ is the conditional probability density of the potential having the value $v_{2}$ at time $t_{2}$, given that $V\left(t_{1}\right)=v_{1}$ and the reset value of the potential after a spike is $v_{0}$. This conditional probability density clearly depends upon $\omega$ and $\phi$, such that in every case the phase $\phi^{\prime}$ associated with a particular time $t^{\prime}$ is given by $\phi^{\prime}=\left[\omega t^{\prime}+\phi\right]_{\bmod 2 \pi}$, where $\phi$ is the initial phase (at time $t=0$ ). This gives a direct and straightforward correspondence between times, $t$, and phases, $\phi$, of the input. Note that the above expression, equation(11), makes no assumptions about the stationarity of the conditional probability density (i.e., it does not require time-translational invariance).

The conditional probability density of the potential in response to noisy periodic input is evaluated in a similar manner to that given above for the probability density [3], and it is also a function of the initial phase of the inputs. The conditional output spike density then follows from equation( 11 ). This enables the phase transition matrix to be calculated, which relates the phase at which the output spike is generated to the initial phase of the inputs [19]. In order to find the interspike interval density (ISI histogram) $\rho(t ; \omega)$ and the period histogram for the neural response to ongoing periodic input generated by inhomogeneous Poisson inputs with period $\omega$, it is necessary to form the appropriate average over the initial phases $\phi$ of the conditional first passage-time densities [19]

$$
\rho(t ; \omega)=\int_{0}^{2 \pi} d \phi f_{\theta}(t ; \omega, \phi) \chi^{(s)}(\phi)
$$

where $\chi^{(s)}(\phi)$ is the stationary distribution of phases evaluated by using the leading eigenvector of the phase transition matrix [19]. The dependence of the synchronization index of the neural response upon the number and amplitude of the synaptic inputs, their synchronization and frequency, and the membrane time constant, are examined. 


\section{Results}

The typical ISI distribution we obtain is plotted in Figure 1 and shows the characteristic multimodal response observed in recording from auditory neurons [10]. The amplitudes of the individual EPSPs in this figure are given by $a=\theta / 16$, where $\theta$ is the difference between the threshold and reset values of the potential. Frequency, $\omega$, and rate, $\lambda$, are measured here in units of the time constant of the membrane, $\tau$, i.e., a frequency (rate) of 1.0 corresponds to one cycle (spike) per $\tau$ units of time.

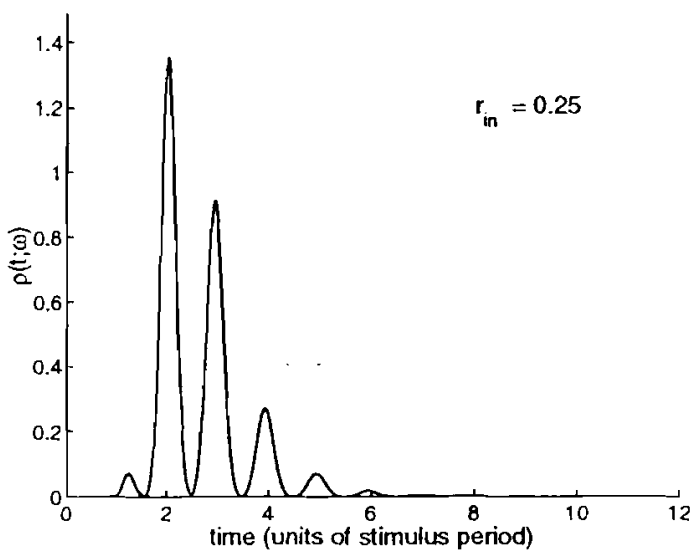

Fig. 1. Plot of the interspike interval distribution, $\rho(t ; \omega)$, for $N=32$ inputs, an input frequency of $\omega=0.3$, input rate of $\vec{\lambda}_{\text {in }}=0.3$, and the amplitudes of the individual EPSPs $a=\theta / 16$, where $\theta$ is the difference between the threshold and reset values of the potential. Frequency and rate are given as cycles (spikes) per unit of $\tau$.

The dependence of the synchronization index upon the frequency is illustrated in Figure 2, which shows that the synchronization index decreases for increasing frequencies. The average rate of the inputs, $\bar{\lambda}_{\text {in }}$, is the same as the frequency in all cases, i.e., there is on average one incoming spike per fibre per cycle of the stimulus. For each value of $N$ the amplitude of the individual postsynaptic potentials is given by $a=2 \theta / N$. The synchronization index of the output spikes is large for all cases in this plot and closest to one when the number of inputs is greatest (the case $N=128$ in Figure 2) Note that the synchronization index of the sinusoidal input here is 0.25 , so that the outputs represent an enhancement of the synchronization relative to the input and this enhancement is more pronounced for larger numbers of inputs and lower frequencies.

In Figure 3 the dependence of the synchronization index upon the degree of modulation of the input, $r_{i n}$, is illustrated for a frequency of $\omega=0.5$ and an average

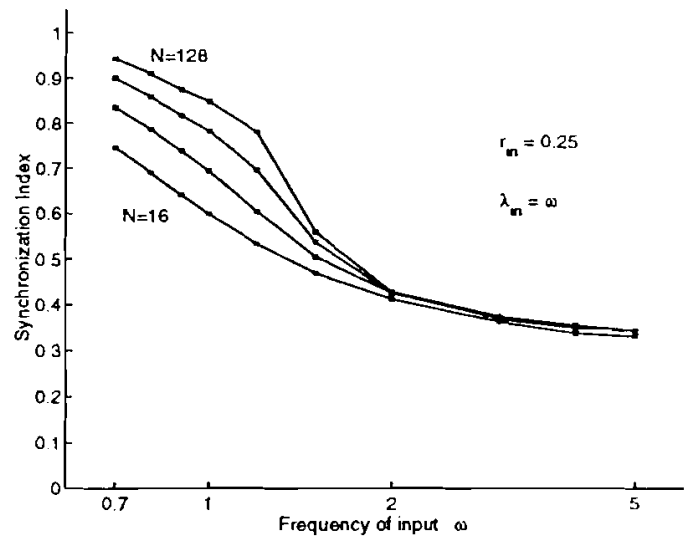

Fig. 2. The synchronization index is plotted against the frequency, $\omega$, (measured in units with $\tau=1$ ), for a value of the input synchronization index $r_{\text {in }}=0.25$. The amplitude of individual EPSPs is $a=2 \theta / N$. The average input rate, $\bar{\lambda}_{\text {in }}$, is the same as the frequency in all cases and the numbers of inputs on the plot are $N=16,32,64,128$.

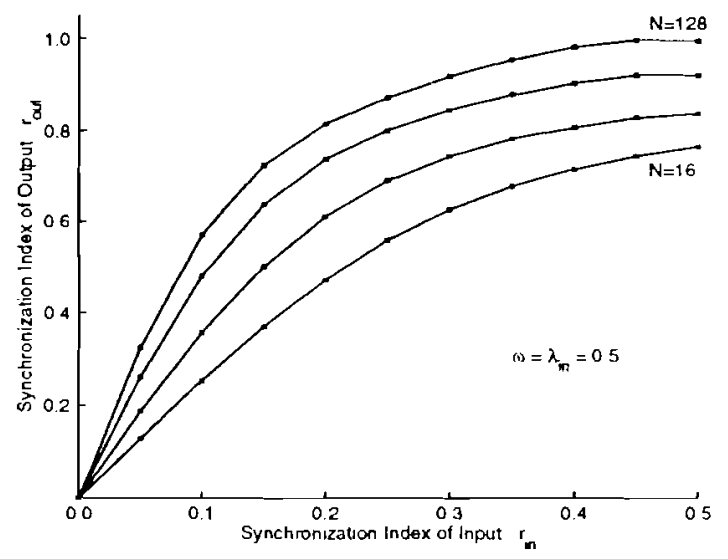

Fig. 3. The synchronization index is plotted as a function of the synchronization index of the input, $r_{i n}$, for a frequency of $\omega=1.0$ and average input rate $\bar{\lambda}_{\text {in }}=1.0$ (in units with $\tau=1)$. The amplitude of individual EPSPs is $a=\theta / N$. The number of inputs on the plot are $N=16,32,64,128$ (bottom to top plots respectively)

input rate of $\bar{\lambda}_{i n 1}=0.5$ (i.e., one spike per input fibre per cycle). The synchronization index of the input is a linear function of the input synchronization index, $r_{\text {in }}$. and varies from 0.5 to zero, so that all points on the plot show an enhancement of synchronization, which is more pronounced for large numbers of inputs. 


\section{Discussion}

This study extends that of earlier studies of coincidence detection and the neural response to noisy periodic synaptic input in a number of important ways. Many of the earlier examinations of the question of coincidence detection were numerical, in which the response to a train of spike inputs was simulated, either using a threshold model with a shot-noise response [8], [6], [14], or a membrane-conductance model [23], [22]. The results of such simulations are, of course, subject to statistical uncertainties in the accuracy of the results obtained and frequently involve massive computational resources, which limits the parameter space that can be explored. Nevertheless, such studies have been instrumental in establishing many of the central features of the neural response, such as the importance of the convergence of inputs to the postsynaptic neuron and the effect of the coincident arrival of the inputs [14]. A comprehensive analytical study of the effect of noisy periodic spike input to an integrate and fire neuron has been carried out [15], in which the relationship between the input and output rates over the range of input synchronization is analyzed and the conditions under which a neuron can act as a coincidence detector are identified by analyzing the coherence gain and the quality factor for coincidence detection. Their analysis, however, centered upon the rate at which output spikes were generated and did not give any detailed information on the timing of individual output spikes. The results presented here extend their study by providing an analysis of the distribution of output spikes. Our analysis enables us to find the interspike interval distribution of the output spikes, the synchronization index of the outputs, and the phase distribution of the outputs.

There are a number of approximations in the analysis presented here whose effect requires closer examination. The integrate and fire model has both strengths as well as shortcomings (see [16] for a review), such as the lack of specific currents that underlie spiking and the linear nature of the summation of the postsynaptic responses. The model therefore does not incorporate any of the postulated nonlinear effects in the dendritic tree [24], nor is it capable of modeling the dynamic nature of synapses [26], [1]. The Gaussian approximation used in this analysis requires that the amplitude of the individual PSPs be small, since the higher order terms in equation(7) (and the corresponding equation for the conditional probability density) are neglected. This is a reasonable approximation in situations where many EPSPs are required to reach threshold. The expression for the conditional first-passage time density is exact for situations where the inputs are uncorrelated, but may introduce errors when the presynaptic spikes are not generated by an (inhomogeneous) Poisson process or the synaptic currents have a finite duration (i.e., when more general synaptic response functions are used, such as those that include finite rise times of the postsynaptic: response). However, it is straightforward to extend the analysis presented here to other forms of periodically varying input, such as the sum of Gaussian distributions [15].

\section{Conclusion}

In conclusion, this study examines the relationship between the synchronization index of noisy oscillating synaptic input and the output spikes that are generated. Previous studies have been concerned mainly with the rate at which output spikes have been generated rather than their timing characteristics [15]. The effect upon the spike output (which itself acts as input to subsequent stages of processing) of both the periodicity of the input and the neural parameters of the leaky integrate and fire neuron upon the propagation of the synchronization and periodicity of the input has been examined, thus illuminating the domain over which temporal information may be transmitted in nellral systems.

\section{References}

[1] L. F. Abbott, J. A. Varela, K. Sen, and S. B. Nelson. Synaptic depression and cortical gain control. Science, 275:220$224,1997$.

[2] S. Anagai, M. A. Friedman, and C. D. Hopkins. Timecoding in the mid-brain of mornyrid electric fish I: Physiclogy and anatony of cells in the nucleus exterolateralis pars anterior. J. Comp. Physiol. A, 182:115-130, 1998.

[3] A. N. Burkitt and G. M. Clark. Analysis of integrate-and-fire neurons: Synchronization of synaptic input and spike output in neural systems. Neural Comput., 11:871-901, 1999.

[4] A. N. Burkitt and G. M. Clark. Calculation of interspike intervals for integrate and fire neurons with Poisson distribution of synaptic inputs, 1999. Subnitted for publication.

[5] A. N. Burkitt and G. M. Clark. New technique for analyzing integrate and fire neurons. In Proceedings of the Computational Neuroscience Meeting 1998, 1999. (to appear in Neurocomputing, 1999).

[6] L. H. Carney. Morlelling the sensitivity of cells in the anteroventral cochlear nucleus to spatiotemporal discharge patterns. Phil. Trans. R. Soc. Lond., B336:403-406, 1992.

[7] C. E. Carr and M.A. Friedman. Evolution of time coding systems. Neur. Comput., 11:1-20, 1999.

[8] H. S. Colburn, Y. Han, and C. P. Culotta Coincidence model of MSO responses. Hear. Res., 49:335-346, 1990.

[9] R. Eckhorn, R. Bauer, W. Jordan, M. Brosich, W. Ḱruse, M. Munk, and H. J. Reitboeck. Coherent oscillations: A mechanism of feature linking in the visual cortex? Biol. Cybern, 60:121-1:30, 1988.

[10] G. L. Gerstein and B. Mandelbrot. Randon walk nıodels for the spike activity of a single neuron. Biophys. J., 4:41-ti8, 1964.

[11] J. M. Goldberg and P. B. Brown. Response of binaural neurons of dog superior olivary comples to dichotic tonal stim- 
uli: Some physiological mechanisms of sound localization. J. Neurophysiol., 32:613-636, 1969.

[12] C. M. Gray, P. König, A. K. Engel, and W. Singer. Oscillatory responses in cat visual cortex exhibit inter-columnar synchronization which reflects global stimulus properties. Nature, 338:334-337, 1989.

[13] D. H. Johnson. The relationship between spike rate and synchrony in responses of auditory-nerve fibers to single tones. J. Acoust. Soc. Am. 68:1115-1122, 1980.

[14] P. X. Joris, L. H. Carney, P. H. Smith, and T. C. T. Yin. Enhancement of neural synchronization in the anteroventral cochlear nucleus. I.responses to tones at the characteristic frequency. $J$. Neurophysiol, 71:1022-1036, 1994.

[15] R. Kempter, W. Gerstner, J. L. van Hemmen, and H. Wagner. Extracting oscillations: Neuronal coincidence detection with noisy periodic spike input. Neural Comput., 10:1987$2017,1998$.

[16] C. Koch. Biophysics of Computation: Information Processing in Single Neurons. Oxford Uni Press, Oxford, 1999.

[17] C. Köppl. Phase locking to high frequencies in the auditory nerve and cochlear nucleus magnocellularis of the barn owl. J. Neurosci., 17:3312-3321, 1997.

[18] A. Papoulis. Probability, Random Variables, and Stochastic Processes. McGraw-Hill International Editions, Singapore, 1991. 3rd Edition.

[19] H. E. Plesser and T. Geisel. Bandpass properties of integrate-fire neurons. In Proceedings of the Computational Neuroscience Meeting 1998, 1998. (to appear in Neurocomputing, 1999).

[20] H. E. Plesser and S. Tanaka. Stochastic resonance in a model neuron with reset. Phys. Lett. A, 225:228-234, 1997.

[21] J. E. Rose, J. F. Brugge, D. J. Anderson, and J. E. Hind. Phase-locked response to low-frequency tones in single auditory nerve fibers of the squirrel monkey. J. Neurophysiol., 30:769-793, 1967.

[22] J. S. Rothman and E. D. Young. Enhancement of neural synchronization in computational models of ventral cochlear nucleus bushy cells. Auditory Neurosci., 2:47-62, 1996.

[23] J. S. Rothman, E. D. Young, and P. B. Manis. Convergence of auditory nerve fibers onto bushy cells in the ventral cochlear nucleus: Implications of a computational model. $J$. Neurophysiol., 70:2562-2583, 1993.

[24] W. R. Softky. Sub-nillisecond coincidence detection in active dendritic trees. Neurosci., 58:13-41, 1994.

[25] F. Theunissen and J. P. Miller. Temporal encoding in nervous systems: A rigorous definition. J. Comput. Neurosci., 2:149-162, 1995.

[26] M. V. Tsodyks and H. Markram. The neural code between neocortical pyramidal neurons depends on neurotransmitter release probability. Proc. Natl. Acad. Sci. USA, 94:719-723, 1997.

[27] H. C. Tuckwell. Introduction to Theoretical Neurobiology: Volume 1, Linear Cable Theory and Dendritic Structure. Cambridge University Press, Cambridge, 1988.

[28] C. von der Malsburg and W. Schneider. A neural cocktailparty processor. Biol. Cybern., 54:29-40, 1986. 


\section{University Library}

\section{- M M I E E R VA A gateway to Melbourne's research publications}

Minerva Access is the Institutional Repository of The University of Melbourne

Author/s:

Burkitt, A. N.;Clark, Graeme M.

Title:

Synchronization of the neural response to noisy periodic synaptic input

Date:

1999

Citation:

Burkitt, A. N., \& Clark, G. M. (1999). Synchronization of the neural response to noisy periodic synaptic input. In Proceedings of the Joint Conference on Neural Networks, Washington.

Persistent Link:

http://hdl.handle.net/11343/27536 